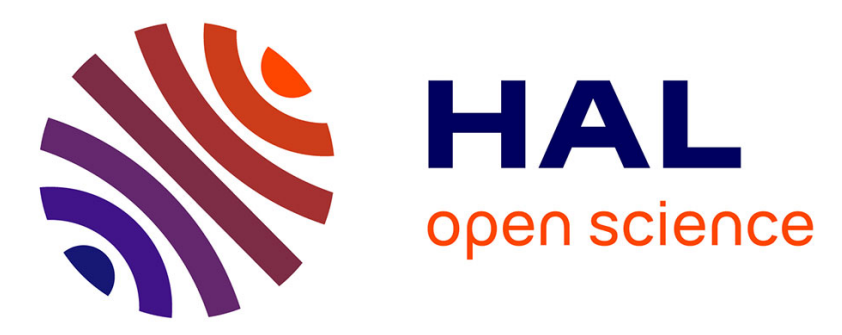

\title{
D'un curriculum auto-prescrit à des curriculums co-produits : le cas de l'enseignement intégré de science et technologie au collège
}

\author{
Maryline Coquidé-Cantor, Corinne Fortin, Christophe Lasson
}

\section{To cite this version:}

Maryline Coquidé-Cantor, Corinne Fortin, Christophe Lasson. D'un curriculum auto-prescrit à des curriculums co-produits: le cas de l'enseignement intégré de science et technologie au collège. Spirale - Revue de Recherches en Éducation , 2013, 52 (1), pp.9-33. 10.3406/spira.2013.1059 hal-01979367

\section{HAL Id: hal-01979367 https://hal.science/hal-01979367}

Submitted on 12 Jan 2019

HAL is a multi-disciplinary open access archive for the deposit and dissemination of scientific research documents, whether they are published or not. The documents may come from teaching and research institutions in France or abroad, or from public or private research centers.
L'archive ouverte pluridisciplinaire HAL, est destinée au dépôt et à la diffusion de documents scientifiques de niveau recherche, publiés ou non, émanant des établissements d'enseignement et de recherche français ou étrangers, des laboratoires publics ou privés. 


\title{
D'un curriculum auto-prescrit à des curriculums co-produits : le cas de l'enseignement intégré de science et technologie au
} collège

\author{
Maryline Coquidé-Cantor, Corinne Fortin, Christophe Lasson
}

\section{Résumé}

L'essai d'un Enseignement Intégré de Science et de Technologie (au collège, en France, a pour objectif de modifier l'organisation de disciplines scientifiques et techniques distinctes (sciences de la vie et de la Terre, sciences technologie) en un enseignement désigné comme intégré. Dans un cadre problématique didactique du curriculum (Martinand 2003a, 2012), nous analysons in situ la dynamique la construction curriculaire et la mise en oeuvre d'EIST par les équipes enseignantes deux collèges. Les résultats obtenus montrent que, s'il se joue dans la tentative d'EIST possible reconfiguration du curriculum de l'enseignement scientifique et technologique, deux cas étudiés montrent plutôt une juxtaposition de contributions disciplinaires.

\section{Abstract}

The test of an Integrated Teaching of Science and Technology (EIST) in some French middle school aims to change the organization of distinct scientific and technical school disciplines (biology and earth sciences, physical sciences, technology). With a didactical theorisation of the curriculum (Martinand 2003a, 2012), we analyze in situ the dynamic curriculum design and implementation of EIST by teaching teams of two colleges. The two case studies show rather a juxtaposition of disciplinary contributions than integration.

\section{Citer ce document / Cite this document :}

Coquidé-Cantor Maryline, Fortin Corinne, Lasson Christophe. D'un curriculum auto-prescrit à des curriculums co-produits : le cas de l'enseignement intégré de science et technologie au collège. In: Spirale. Revue de recherches en éducation, $n^{\circ} 52,2013$. L'enseignement intégré de science et de technologie (EIST) au collège : à la recherche d'un curriculum. pp. 9-33;

doi : https://doi.org/10.3406/spira.2013.1059

https://www.persee.fr/doc/spira_0994-3722_2013_num_52_1_1059

Fichier pdf généré le 31/03/2018 


\title{
D'UN CURRICULUM AUTO-PRESCRIT À DES CURRICULUMS CO-PRODUITS
}

\author{
LE CAS DE L'ENSEIGNEMENT INTÉGRÉ \\ DE SCIENCE ET TECHNOLOGIE AU COLLÈGE
}

\begin{abstract}
Résumé : L'essai d'un Enseignement Intégré de Science et de Technologie (EIST) au collège, en France, a pour objectif de modifier l'organisation de disciplines scolaires scientifiques et techniques distinctes (sciences de la vie et de la Terre, sciences physiques, technologie) en un enseignement désigné comme intégré. Dans un cadre problématique de didactique du curriculum (Martinand 2003a, 2012), nous analysons in situ la dynamique de la construction curriculaire et la mise en œuvre d'EIST par les équipes enseignantes de deux collèges. Les résultats obtenus montrent que, s'il se joue dans la tentative d'EIST une possible reconfiguration du curriculum de l'enseignement scientifique et technologique, les deux cas étudiés montrent plutôt une juxtaposition de contributions disciplinaires.

Mots-clés : discipline scolaire, dispositif, curriculum, science, technologie, transformation curriculaire, intégration, indifférenciation, juxtaposition.

Les domaines d'étude relatifs à l'éducation scientifique et technologique, à l'école primaire et au secondaire inférieur en France (école moyenne), se présentent actuellement dans plusieurs domaines scolaires successifs, avec une compartimentation disciplinaire progressive : Découverte du monde (matière, vivant, objets et matériaux) en cycle 1 et en cycle 2, Sciences expérimentales et technologie du cycle 3 de l'école primaire, organisation en différentes disciplines Sciences de la Vie et de la Terre, Physique-Chimie, et Technologie au collège. Face à ce qui pouvait apparaître comme un cloisonnement trop étanche de l'enseignement secondaire, des modifications de curriculum ont été avancées depuis deux décennies, principalement sous forme de dispositifs pluridisciplinaires valorisant les actions coordonnées des enseignants. Elles s'inscrivent dans le projet de constitution de pôles disciplinaires, impulsé par le Conseil National des Programmes depuis le milieu des années 1990 (Ferry 1995). Dans ce cadre, un essai d'Enseignement Intégré de Science et de Technologie (EIST) est organisé en France depuis 2006, à l'initiative de l'Académie des Sciences et de l'Académie des Technologies, en partenariat avec le Ministère de l'Éducation Nationale, principalement en classe de $6^{\mathrm{e}}$ et dans quelques classes de cinquième. Cette tentative institutionnelle promeut une démarche pédagogique «d'investigation », comme le préconise le Rapport Rocard pour la commission européenne (2007), et une modification du curriculum, visant à améliorer la transition entre l'école et le collège, et à augmenter l'attractivité des sciences et de la technologie auprès des élèves.
\end{abstract}

Spirale - Revue de Recherches en Éducation - $2013 N^{\circ} 52$ (9-33) 
Après avoir rappelé diverses modifications récentes de l'enseignement scientifique et technologique, nous comparerons différentes logiques d'élaboration curriculaire. Le cadre problématique de didactique du curriculum (Martinand 2003a, 2012, 2013) permettra ensuite d'analyser la dynamique in situ de la construction curriculaire et de la mise en œuvre d'EIST par les équipes enseignantes de deux collèges.

\section{DES CHANGEMENTS CURRICULAIRES DE L'ENSEIGNEMENT SCIENTIFIQUE ET TECHNOLOGIQUE À L'ÉCOLE MOYENNE EN FRANCE}

Les essais de transformations curriculaires actuelles de l'enseignement scientifique et technologique en France semblent ouvrir un débat ancien. Est-il utile, au collège, de commencer par des disciplines constituées, enseignées par des enseignants spécialistes ayant une formation spécifique ? Cet objectif d'intégration sera-t-il mieux assuré par un professeur unique de science, ou bien par plusieurs professeurs spécialisés (en Sciences de la Vie et de la Terre, en Sciences Physiques, en Technologie) qui coordonnent leurs enseignements ? Le sujet est très controversé et non dénué d'arrière-plans financiers et administratifs qui paralysent la réflexion sereine. Le temps qui est nécessaire à la coordination d'une équipe enseignante représente, par exemple, un frein important.

\subsection{Des dispositifs et des modifications curriculaires}

Lebeaume $(2002,2008,2009)$ a analysé différentes tentatives de modifications curriculaires de l'enseignement scientifique par les institutions éducatives françaises, depuis plusieurs décennies et favorisant l'interdisciplinarité. L'expérience des «classes nouvelles» (1945-1961) fut ainsi une innovation radicale. Inspirée par Dewey, elle portait tout à la fois sur les contenus, la relation pédagogique et l'organisation scolaire. Chaque classe nouvelle était prise en charge par une équipe pédagogique, avec des projets relatifs à des problèmes de vie reconnus par les élèves et exigeant une approche interdisciplinaire (Lebeaume 2008). Des moyens spécifiques étaient attribués, en particulier du temps pour la concertation. Pour l'enseignement scientifique, la pédagogie favorisait le développement de l'apprentissage par résolution de problèmes et le décloisonnement des apprentissages par l'étude du milieu. L'expérience de ces classes nouvelles a pris une grande extension ${ }^{1}$ et elle absorbait des crédits importants, principalement en créations de postes et en stages de formation. Débordé par les problèmes quantitatifs, le ministère abandonna cette expérience en 1961. Après l'abandon de cette expérience des classes nouvelles, une forme d'enseignement intégré fut créée avec les Travaux Scientifiques Expérimentaux (TSE), mis en œuvre de 1961 à 1968, à raison d'une heure dans le cycle d'observation (Lebeaume 2002, 2009). Le but recherché était l'acquisition de la capacité, par les élèves, à conduire librement une recherche expérimentale ou documentaire. Ces activités déroutaient cependant plusieurs enseignants car elles nécessitaient parfois des connaissances non acquises à l'université. Elles ne donnaient pas lieu, par ailleurs, à une évaluation globale, ni à la création d'un corps d'inspecteurs spécialisés et responsables. L'innovation des

\footnotetext{
${ }^{1}$ Environ 16000 élèves en 1948.
} 
TSE fut abandonnée dans les réformes de 1969. Par la suite, l'Institut National de Recherche Pédagogique (INRP) a développé des recherches sur l'introduction de l'interdisciplinarité. Elles se sont développées jusqu'en 1980 dans les lycées, puis dans les collèges. Diverses innovations ont exploré des formes d'interdisciplinarité dans le cadre de l'enseignement scientifique à l'école moyenne et les difficultés se sont révélées nombreuses. Elles n'ont pas toutes été bien étudiées encore et mériteraient une reprise d'analyse historique des contextes, des organisations et des choix pédagogiques.

\subsection{Depuis les années deux mille}

Le collège français accueille aujourd'hui, dans un cadre unique, tous les élèves à l'issue de l'école élémentaire. Les enseignements du second degré, organisés en disciplines scolaires dont les contenus sont définis par des programmes nationaux obligatoires, sont pris en charge par des professeurs spécialisés, ayant reçu tout d'abord une formation académique puis une formation pédagogique et didactique spécifique disciplinaire. Depuis les années 2000, les orientations de la politique éducative tendent à introduire, dans l'organisation du collège unique, des «bougés », avec des glissements ou des déplacements du projet curriculaire et de l'organisation (Dutercq et Derouet 2004). Les disciplines du collège ont été particulièrement visées : par des demandes d'ouverture, des nouvelles prescriptions et des dispositifs pédagogiques, organisés dans la classe ou entre classes, et à durée de vie plus ou moins grande. Des mesures ou des innovations institutionnelles ont ainsi impulsé la mise en œuvre de dispositifs, tels que les parcours diversifiés, les travaux croisés ou les itinéraires de découverte. Ils valorisent les usages des technologies de l'information et de la communication, l'éclatement des regroupements par classes, les activités «pluri » ou «interdisciplinaires » et le travail d'équipe (Lessard et Barrère 2005). Les parcours diversifiés (1996-2005), par exemple, n'étaient pas soumis à des cadres stricts d'horaires et de programmes. Incitant à un travail en équipe des enseignants, les approches thématiques préconisées avaient à favoriser un lien entre les savoirs scolaires et la connaissance du monde contemporain, tandis que les tâches scolaires concernaient des objets non encore disciplinaires et visaient davantage le développement de compétences (Coquidé 2001). Des études sur un autre dispositif interdisciplinaire, les itinéraires de découverte, montraient cependant la fragilité d'une organisation qui prenait en compte uniquement des questions pédagogiques, avec une cohérence que Lebeaume et Magneron (2004) ont qualifié « de surface ».

D'autres modifications curriculaires, introduites depuis 2006, tendent au rapprochement des disciplines d'enseignement scientifique et technique (Sciences Physiques, Sciences de la Vie et de la Terre, Technologie et Mathématiques) (Coquidé 2008). Ainsi, le Socle commun de connaissances et de compétences (MEN 2006) traite de ces quatre disciplines dans un même pilier et il introduit des thèmes de convergence, ce qui devrait conduire, plus que jamais, à une nécessité de grande coordination des enseignements.

Un aperçu historique de l'enseignement scientifique, à l'école moyenne en France, montre une évolution de l'organisation curriculaire, structurée alternativement selon des modalités de fermeture avec une stricte compartimentation disciplinaire, ou d'ouverture avec divers dispositifs pédagogiques. Dans une perspec- 
tive sociale, de nouvelles missions éducatives sont attribuées à l'école, par exemple l'éducation à la santé ou au développement durable. Ces deux mouvements se conjuguent pour une collaboration ou une convergence des disciplines, autour de dispositifs ou d'essais pédagogiques visant davantage d'intégration. Ces tendances ont des incidences fortes sur la pratique attendue des professeurs, avec une incitation à des modalités collectives de travail, désignées sous les termes de co, pluri, multi ou interdisciplinarité. Nous avons accès, cependant, le plus souvent uniquement aux «traits de surface» (Lebeaume 2009) des transformations curriculaires (les programmes, les recommandations, les dispositifs d'enseignement,...). Ils sont les résultats visibles d'un processus complexe, avec des logiques épistémologiques ou des logiques d'action, profondes et moins visibles, soumises à différentes tensions, didactiques, sociales, économiques ou politiques.

\section{DES LOGIQUES DE CONSTRUCTIONS CURRICULAIRES DIFFÈRENTES}

\subsection{Différentes tensions d'élaboration curriculaire}

Les visées et l'élaboration d'un curriculum sont en effet source de tensions. Lenoir, Larose et Lessard (2006) suggèrent ainsi quatre axes pour l'analyse de structuration curriculaire :

- l'ordonnancement du contenu : du curriculum intégré au curriculum compartimenté (selon la distinction initiale de Bernstein (1975) entre «code intégré » et « code série ») ;

- la centration du curriculum : sur les objets d'apprentissage (subject-centered model) ou bien sur les sujets (child-centered pattern);

- les finalités éducatives ;

- les modalités opérationnelles (dont les conceptions psychopédagogiques de l'apprentissage, les tâches scolaires privilégiées et les méthodes pédagogiques).

Ces quatre axes d'analyse peuvent être rapprochés de la distinction proposée par Ross (2000). À partir de l'analyse critique sociohistorique de différents curriculums anglais, Ross mobilise une métaphore de différents jardins pour distinguer quatre types d'élaboration curriculaire : les élaborations content-driven (le jardin compartimenté et strictement ordonné Baroque garden), objectives-driven (le jardin potager utilitaire Dig-for-victory garden), process-driven (le jardin à l'anglaise paysager Landscape garden), qu'il rapproche d'autres analyses (tableau 1). Ces trois orientations concernent les types de pilotage de chaque curriculum : selon des objectifs à atteindre (objectives-driven), selon des contenus à rencontrer (content-driven) ou selon des expériences formatrices à vivre (process-driven). Les moyens de mise en œuvre et les évaluations adéquates de chacun de ces types curriculaires ne peuvent être les mêmes.

Sur un plan politico-idéologique, Ross (2000) précise des rivalités idéologiques et des alliances possibles entre les conceptions modélisées de ces trois types de curriculums. Ainsi, les curriculums content-driven et objectives-driven se rapprochent par une perspective d'un champ d'apprentissage fixé à l'avance, les curriculums objectives-driven et process-driven par un souci d'égalitarisme et une 
forte responsabilité des élèves, tandis que les curriculums process-driven et content-driven convergent par rapport à une antipathie au marché ou à toute forme d'instrumentalisation ${ }^{2}$.

\begin{tabular}{|l|l|l|l|}
\cline { 2 - 4 } \multicolumn{1}{c|}{} & \multicolumn{1}{c|}{$\begin{array}{c}\text { Content-driven } \\
\text { Baroque garden }\end{array}$} & $\begin{array}{c}\text { Objectives-driven } \\
\text { Dig-for-victory garden }\end{array}$ & \multicolumn{1}{|c|}{$\begin{array}{c}\text { Process-driven } \\
\text { Landscape garden }\end{array}$} \\
\hline Blyth (1967) & Preparatory & Elementary & Developmental \\
\hline Lawton et alii (1976) & $\begin{array}{l}\text { Subject-centered } \\
\text { Knowledge-centered }\end{array}$ & Society centered & Child-centered \\
\hline Goodson (1987) & Academic & Utilitarian & Pedagogic \\
\hline Golby (1989) & Liberal humanist & Technocratic & $\begin{array}{l}\text { Child-centered progres- } \\
\text { sivism }\end{array}$ \\
\hline
\end{tabular}

Tableau 1 : Différents « types » curriculaires, adaptation d'après Ross $(2000,98)$

\subsection{Intégration, interdisciplinarité ou indifférenciation?}

Une tension importante est «pour qui y a-t-il intégration, interdisciplinarité ou indifférenciation disciplinaire ?». Elle relève de perspectives qui différent, selon le point de vue institutionnel, de la recherche, des enseignants ou des élèves.

D'autres tensions peuvent relever des possibilités de flexibilité et de la plus ou moins grande souplesse laissée à l'enseignant. Ceci permet de considérer les caractéristiques d'un curriculum avec une perspective d'intégration, dès son élaboration, des domaines d'étude (ou curriculum intégré), par rapport à un curriculum avec des domaines distincts (curriculum compartimenté de Bernstein) et des dispositifs pédagogiques qui visent à favoriser un processus d'intégration (ou curriculum intégrateur), et les mouvements de passage de l'un à l'autre.

Cette distinction entre curriculum « compartimenté » (ou strictement disciplinaire) et curriculum «intégré » (ou adisciplinaire) est également mentionnée par Loepp (1999), qui envisage différentes mises en œuvre d'enseignement intégré. Pour préciser le sens des termes intégration et interdisciplinarité, qui sont souvent confondus, Loepp avance une métaphore culinaire. Contrairement aux tranches uniformes d'une génoise, qui peuvent représenter une approche juxtaposée des enseignements disciplinaires (avec éventuelle interdisciplinarité), le gâteau marbré, intégrant en une seule pâtisserie gâteau au chocolat et gâteau à la vanille, permet de situer l'approche intégrée des enseignements autour d'un thème ou d'un problème de réalisation (adisciplinaire).

Concernant «l'intégration », un ensemble d'interrogations apparaît donc récurrent pour la recherche. Un travail d'intégration, de structuration, d'unification doit-il être présenté au départ comme une condition de possibilité de l'assimilation du savoir scientifique dans un curriculum intégré ? Ou bien ce travail d'intégration doit-il être considéré en accompagnement, comme un but final de l'enseignement, dans un processus intégrateur? Comment faire converger, mettre en relation, ou faire éclater des disciplines scolaires? Des injonctions brutales mettent en avant le transversal pour rechercher à travers les disciplines les capacités, les

${ }^{2}$ Si l'on reprend la distinction proposée par Ross, l'introduction en France des perspectives de Socle commun de connaissances et de compétences au collège induisent des tensions de réorganisation entre un curriculum content-driven et un curriculum competency-driven. 
attitudes, les méthodes, les compétences ou les performances, qui sont supposées communes à plusieurs disciplines scolaires et détachables d'un contenu précis (Rumelhard et Desbeaux-Salviat 2000). Elles semblent négliger le va-et-vient indispensable entre spécialisation et intégration. L'interdisciplinarité peut certes favoriser une véritable rencontre, mais non dénuée d'ambigüité (Lenoir 2006). Quant à une tentative de décloisonnement ou de reconfiguration des disciplines scientifiques et technologiques, celle-ci ne peut se décréter comme ce qui serait, $a$ priori, commun aux différentes disciplines. Une réflexion pour une unification ou intégration des enseignements, peut ainsi envisager différentes organisations :

- des dispositifs ou des démarches incitant à de la codisciplinarité (Chevallard 2004), pluri ou interdisciplinarité, encourageant les interactions ou la coordination entre des enseignants spécialistes pour un processus d'intégration ;

- des organisations curriculaires qui envisagent plus d'intégration a priori des enseignements, en particulier pour des enseignants davantage polyvalents (Baillat et Niclot 2003) ;

- un report de la spécialisation et de la compartimentation, en maintenant une forme d' « indifférenciation » du domaine scientifique et technique, comme au primaire, avec un report temporel de la visée de disciplinarisation.

\subsection{Différentes mises en æuuvre d'enseignement intégré}

La mise en place d'un enseignement intégré est néanmoins difficile. Wallace, Sheffield, Rennie et Venville (2007) proposent une analyse de différentes études, conduites ces dix dernières années dans des écoles moyennes australiennes. Ils tentent ainsi d'analyser des échecs et des décalages, entre les prescriptions d'intégration curriculaire et les mises en œuvre effectives.

Pour Loepp (1999), un enseignement intégré implique des changements importants : des contenus et des modalités d'évaluation, mais aussi des représentations sociales (Jodelet 1989) de l'ensemble de la communauté éducative. Il compare différentes mises en œuvre de curriculums intégrés, conçus autour de thèmes pour de jeunes élèves et à partir de problèmes pour des élèves plus âgés. L'interdisciplinary model s'appuie sur l'organisation d'un emploi du temps ménageant des moments de travail à plusieurs enseignants. Le problem-based model est centré sur la résolution du problème et différentes disciplines apportent leur contribution. Le theme-based model est organisé par des objectifs susceptibles d'être abordés par chacune des disciplines. Loepp met en évidence un problème majeur d'articulation de ces modalités d'enseignement avec des programmes disciplinaires ou bien des standards (Mons et alii 2006, Dell'Angelo et alii 2011). Tout comme Wallace et alii, il conclut qu'une perspective d'intégration curriculaire représente une réforme profonde de l'enseignement, et il identifie plusieurs implications pour que les modalités d'une telle réforme d'enseignement intégré soient reconnues. Les principales concernent une nécessité pour les professeurs d'avoir une conception constructiviste des apprentissages, de se sentir membre d'une communauté éducative, le besoin d'une formation professionnelle ${ }^{3}$, une exigence de nouvelles ressources pour l'enseignement et une obligation de l'association des parents. Face à ces difficultés d'articulation, nous élargirons, plus loin, la réflexion sur les

\footnotetext{
${ }^{3}$ Formation sur les fondements de ces modalités, sur l'organisation pédagogique des travaux de groupes, pour de nouvelles modalités d'évaluation...
} 
contenus d'enseignement, sur leurs organisations (disciplinaires ou non) et sur le curriculum formant un système.

\subsection{Diverses tensions dans le cas de l'essai d'EIST}

Et dans le cas de la tentative d'EIST ? Quelle(s) élaboration(s) et quelles mises en oeuvre sont envisagées ? Dans les textes institutionnels, la proposition d'EIST apparaît comme une forme d'aménagement qui promeut tout à la fois :

- un décloisonnement disciplinaire, en proposant une nouvelle organisation dans laquelle des thématiques traitées (Matière et matériaux, Énergie et énergies...) ne sont pas des objets disciplinaires ;

- le maintien des disciplines, en fléchant à l'intérieur de chacune des thématiques ce qui relève des SVT, de la Technologie et des SPC.

L'objectif affiché «n'est pas de faire disparaître les disciplines » mais de «favoriser la cohérence entre elles et mettre en évidence leur nécessaire synergie » (Guide EIST, 8)

Dans le cas de la tentative d'EIST, la mise en tension entre disciplines scolaires et «vision unifiée » de science (au singulier) et de technologie interroge, en outre, la signification donnée à la conjonction de coordination «et». Une première tension est relative à une compartimentation/décompartimentation, avec une double signification, organisationnelle et épistémologique. L'innovation contemporaine d'EIST se distingue ainsi de l'enseignement des Travaux Scientifiques Expérimentaux évoqués auparavant, mis en place en 1961 pour les classes du cycle d'observation, alors pris en charge par des enseignants bivalents ou par des équipes d'enseignants (Lebeaume 2002, 2008). Une deuxième tension concerne les disciplines et leur hiérarchie, avec des statuts de valorisation/dévalorisation, de disciplines majeures/mineures. Au collège, ces tensions sont manifestes dans le poids des disciplines lors des évaluations. Dans les commentaires des enseignants, pour la mise en œuvre de l'EIST et pour son évaluation, des inquiétudes récurrentes concernant la technologie et son statut sont manifestes. Dans le prolongement des tensions concernant la compartimentation et le statut des disciplines, on peut analyser, dans les documents formels accompagnant l'essai, une autre tension relative aux perspectives d'un enseignement d'EIST. En particulier, comment s'articulent le singulier et le pluriel de cet enseignement de science(s) et de technologie ? S'agit-il de spécifier ou d'unifier, de diversifier ou de réduire ? Que peut signifier une démarche pédagogique uniformisante «d'investigation »?

\section{UN CADRE CONCEPTUEL}

\section{DE DIDACTIQUE DU CURRICULUM}

La tentative d'EIST interroge l'extension et la réorganisation des contenus enseignés en sciences et en technologie, avec de nouveaux contours et des formes de travail enseignant collaboratif ou coopératif (Marcel et alii 2007, Mérini 2005). L'objectif de notre recherche est de décrire les choix et les décisions curriculaires de l'équipe enseignante, lors de la construction et de la mise en œuvre d'un curriculum local effectif au sein de l'établissement. 
3.1. Pour une analyse de dynamique de construction curriculaire d'EIST

Notre analyse ne se place pas d'un point de vue de la sociologie du curriculum (Forquin 2008), un curriculum référent n'étant pas ici le centre organisateur d'un projet institutionnel au même titre que le sont des programmes scolaires, ni dans une approche où le curriculum effectif relèverait d'expériences formatrices de production d'apprentissage (Perrenoud 1993). Nous avons retenu le cadre problématique de Martinand (2003a), qui considère les questions d'élaboration de curriculum en éducation scientifique et technique, celles des mises en oeuvre et celles d'écarts entre curriculums prescrits et réels comme des enjeux théoriques de la construction curriculaire. Pour problématiser cette élaboration et ces mises en œuvre, il avance les concepts de curriculum «prescrit», «produit», «potentiel » et «possible» (Martinand 2003a, 2012) (figure 1).

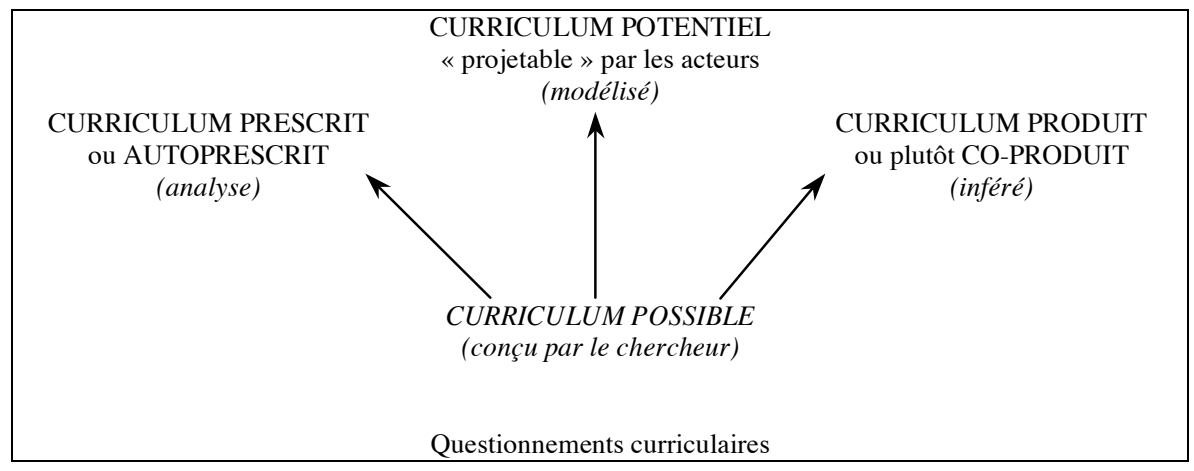

Figure 1 : Les questionnements curriculaires (Martinand 2012)

Le « curriculum prescrit» s'étudie par analyse documentaire et le «curriculum produit » ou «co-produit » (par les enseignants et le élèves), par inférence à partir d'observations, d'enregistrements, d'autoanalyses et confrontations. Le «curriculum potentiel» est une modélisation interprétative, construite par les chercheurs, pour caractériser tout un espace de potentialités de mise en œuvre et expliquer des différences entre prescrit et produit. Ce «curriculum potentiel» représente aussi ce que l'enseignant est capable d'imaginer pouvoir mettre en œuvre d'un curriculum prescrit, dans ses conditions de travail et avec ses élèves. Ces questionnements sur les élaborations curriculaires, les mises en œuvre et les écarts, exigent un « référentiel» pour pouvoir effectuer analyses et comparaisons. Pour Martinand (2013), c'est le «curriculum possible» (ou «des possibles »), conçu par le chercheur, et auquel sont comparés prescrit, produit et potentiel, qui joue cette fonction de référentiel.

Notre interrogation dans l'essai d'EIST a mobilisé concrètement ces questionnements d'élaboration et de mise en œuvre de curriculum. Le curriculum possible suppose de ne pas se laisser pas enfermer dans une seule logique, bipolaire (décloisonnement versus cloisonnement disciplinaire), mais aussi interroger et penser la légitimité et la pertinence de différentes formes (par exemple curriculums disciplinaires ou non, intégrés ou intégrateurs). Quelles sont alors les normes éducatives qui disparaissent ou qui sont introduites? Quelles sont les relations éventuelles à des disciplines et leur nature (autonomie, subordination, interdépen- 
dance...) ? Le curriculum possible interroge ainsi les différentes visées éducatives en termes de contenus, existants ou à inventer, et pas seulement de contenus à organiser (Martinand 2013). De ce point de vue, il engage la responsabilité des chercheurs et nécessite d'expliciter les enjeux éducatifs, scientifiques et sociaux (White 2004). La rationalité scientifique ne peut pas être seule en cause pour justifier d'un contenu. La légitimité sociale des contenus enseignés et celle de leurs mises en œuvre sont aussi à considérer. Le curriculum possible est, par conséquent, un choix parmi d'autres possibles, éclairé par la pluralité des rationalités, en fonction des finalités éducatives choisies ou retenues.

\subsection{Questions de recherche}

Plus précisément, nous nous sommes intéressés ici au processus d'élaboration et de construction curriculaire, par l'équipe enseignante d'EIST. Nous interrogeons l'élaboration d'un curriculum local auto-prescrit, issu du projet éducatif institutionnel présenté dans les Guides EIST, mais relevant surtout des choix et des décisions, des contraintes et des obstacles rencontrés par l'équipe enseignante, pour concevoir et mettre en œuvre son propre projet éducatif en EIST. Nous avons fait l'hypothèse que le curriculum auto-prescrit nécessite, pour chaque enseignant, de faire l'expérience de l'altérité disciplinaire, par la construction d'une culture professionnelle commune, fondée sur une technicité partagée (Martinand 1994) en termes de savoirs et de savoir-faire didactiques, pour donner sens à un projet éducatif commun. Les séances hebdomadaires de concertation, entre les trois enseignants de différentes disciplines de l'équipe EIST, instaurent un espace participatif d'échanges et de négociations, d'où émergent des options et des orientations qui contribuent à l'élaboration de ce curriculum local auto-prescrit.

Cette expérience de l'altérité disciplinaire ne va cependant pas de soi. En effet, la tentative d'EIST est un enseignement en tension qui vise à promouvoir une nouvelle unité d'enseignement tout en maintenant un pluralisme disciplinaire. Dans ces conditions, comment l'équipe enseignante problématise-t-elle ce nouvel enseignement? Quels sont ses choix au regard des recommandations institutionnelles ? Au regard des curriculums possibles envisagés par les chercheurs (paragraphes 2.3, 2.4 et 3.1) ? Les choix relèvent-ils plutôt d'une intégration, d'une juxtaposition ou d'une indifférenciation disciplinaire ? En cas d'intégration, celle-ci se rapproche-t-elle d'interdisciplinary, de problem-based ou bien de theme-based model, selon la modélisation de Loepp (1999) ?

La recherche vise aussi à questionner les écarts entre le curriculum autoprescrit et les curriculums co-produits au niveau de la mise en œuvre, de façon continuée tout au long de l'année scolaire, de chaque enseignement. Y a-t-il un « effet disciplinaire » dans la mise en œuvre du curriculum auto-prescrit EIST, selon la formation et la spécialité disciplinaire de chaque enseignant (technologie, SVT, PC) ?

\subsection{Méthodologie}

Il s'agit d'études de cas. Deux équipes enseignantes ont été suivies dans deux collèges, situés en banlieue de deux grandes métropoles (Coquidé et alii 2011) : 
- le collège A, de petite taille (350 élèves), est situé en $\mathrm{REP}^{4}$. L'équipe enseignante n'a pas demandé à participer à l'essai d'EIST mais elle a répondu favorablement à la demande du chef d'établissement qui souhaitait que le collège $\mathrm{y}$ soit inscrit dès 2006 ;

- le collège $B$, de taille moyenne (561 élèves), possède pour chaque niveau des classes SEGPA ${ }^{5}$ et des classes d'enseignement général. L'équipe enseignante s'est portée volontaire pour une tentative d'EIST en 2009.

Les deux équipes ont assisté au séminaire national EIST $^{6}$ dans le cadre du Plan National de Formation des Enseignants.

3.3.1. Élaboration du corpus : recueil et sélection des données

Les recommandations institutionnelles et les documents d'accompagnement (les guides EIST) représentent une réponse programmatique émanant des institutions (Académie des Sciences et Académie des Technologies, MEN), en termes de contenus d'enseignement et de suggestions pour la mise en œuvre d'EIST.

Les diverses propositions pédagogiques et didactiques, qui sont discutées et négociées par l'équipe en concertation, peuvent être considérées comme contribuant à un curriculum potentiel.

Le curriculum auto-prescrit, élaboré par l'équipe enseignante, résulte des propositions collectivement retenues parmi celles énoncées en concertation. Il relève d'une auto-prescription locale et renseigne sur les choix collectifs de l'équipe pour mettre en œuvre l'EIST. Il résulte de l'interaction et du partage de connaissances expérientielles de chaque enseignant pour envisager, collectivement, un enseignement intégré. Il s'agit, pour l'équipe enseignante, d'investir un nouveau champ, en rupture avec les enjeux éducatifs traditionnels et en l'absence d'une mémoire professionnelle commune, avec sa part d'incertitude et de prise de risque.

Le curriculum co-produit relève de la mise en œuvre, dans chaque classe de «Science et Technologie », de façon continuée tout au long d'une année scolaire, du curriculum auto-prescrit. Il peut renseigner sur un effet disciplinaire dans l'appropriation d'un EIST par chaque enseignant, selon sa formation et sa discipline (technologie, SVT, PC).

Les conditions de recueils des données n'ont pas été identiques pour les deux équipes, en raison de contraintes liées à la disponibilité des enseignants. L'enregistrement vidéo de la concertation pour l'élaboration curriculaire n'a été réalisé qu'avec l'équipe du collège $\mathrm{A}$. Dans le collège $\mathrm{B}$, les séances de concertation pour cette élaboration se sont déroulées avant la rentrée scolaire, pendant les grandes vacances ${ }^{7}$. De même, les données recueillies dans le collège A et B ne portent pas sur des thématiques identiques : l'équipe du collège $\mathrm{A}$ a choisi de tra-

${ }^{4}$ REP : acronyme de Réseau d'Éducation Prioritaire

${ }^{5}$ SEGPA : acronyme de Section d'Enseignement Général et Professionnel Adapté (SEGPA)

${ }^{6} \mathrm{Ce}$ séminaire est organisé pour guider les enseignants et favoriser les échanges entre enseignants néophytes et enseignants déjà impliqués dans l'EIST.

La concertation hebdomadaire durant l'année scolaire a alors principalement été consacrée à la mise en page des fiches activité-élèves ou à tester du matériel ou des instruments (microscope, perceuse, etc.) utilisés en classe. 
vailler sur «Énergie et énergies », tandis que celle du collège B sur « Matière et matériaux».

3.3.2. Analyse du corpus

La grille d'analyse (tableau 2) vise à caractériser des indicateurs des modes d'intégration d'EIST retenus.

\begin{tabular}{|c|c|c|}
\hline Recueil de données & Corpus & Analyse du corpus \\
\hline Documents écrits & $\begin{array}{l}\text { Guide découverte EIST et } \\
\text { guide « Matière et matériaux » } \\
\text { pour la classe de } 6^{\mathrm{e}}\end{array}$ & $\begin{array}{l}\text { Organisation curriculaire envisagée } \\
\text { Quelles articulations des disciplines scien- } \\
\text { tifiques et de la technologie? }\end{array}$ \\
\hline $\begin{array}{l}\text { Enregistrements vidéo } \\
(1 \mathrm{~h} 30) \text { en camera fixe } \\
\text { (plan d'ensemble des } \\
\text { trois enseignants en dis- } \\
\text { cussion) }\end{array}$ & $\begin{array}{l}\text { Script des échanges entre les } \\
\text { trois enseignants lors de la } \\
\text { séance de concertation }\end{array}$ & $\begin{array}{l}\text { Projet curriculaire de l'équipe enseignante } \\
\text { Échanges et négociations de l'équipe en- } \\
\text { seignante sur : } \\
\text { - l'intégration science et technologie dans } \\
\text { la progression, dans les activités-élèves, } \\
\text { - les choix collectifs de l'équipe ensei- } \\
\text { gnante }\end{array}$ \\
\hline Productions écrites & $\begin{array}{l}\text { Documents de travail élaborés } \\
\text { et rédigés par les deux équipes. } \\
\text { Contenu et organisation des } \\
\text { leçons élaborées. } \\
\text { Fiche d'activités élèves } \\
\end{array}$ & $\begin{array}{l}\text { Elaboration d'un curriculum auto-prescrit } \\
\text { Choix et prises de décision collectifs sur ce } \\
\text { que les élèves doivent apprendre et sur les } \\
\text { activités à réaliser en classe d'EIST }\end{array}$ \\
\hline $\begin{array}{l}\text { Enregistrements vidéo } \\
\left(90^{\prime}\right) \text { de chaque profes- } \\
\text { seur dans sa classe }\end{array}$ & $\begin{array}{l}\text { Script de ce « que l'enseignant } \\
\text { dit » et « fait » réellement dans } \\
\text { la classe au regard de ce qui } \\
\text { été prescrit par l'équipe ensei- } \\
\text { gnante EIST }\end{array}$ & $\begin{array}{l}\text { Mise en ouvre du curriculum auto-prescrit } \\
\text { en classe et curriculum co-produit } \\
\text { Aménagement du curriculum auto-prescrit } \\
\text { selon la spécialité de l'enseignant en classe } \\
\text { EIST. Gestion des activités élèves }\end{array}$ \\
\hline
\end{tabular}

Tableau 2 : Recueil des données et grille d'analyse du corpus

\section{RÉSULTATS}

4.1. Analyse de recommandations institutionnelles

L'EIST vise à proposer aux élèves une «vision unifiée de la science et de la technologie » (Guide découverte de l'EIST, 23) par une approche «interdisciplinaire », c'est-à-dire « une conception plus globale et cohérente de la science, se déclinant en diverses disciplines étroitement liées par leur méthode commune (la rationalité scientifique), et leur objet commun (le monde et ses phénomènes et l'action de l'homme) » (Guide découverte de l'EIST, 24). L'objectif est « de proposer des situations (...), pour aider les élèves à élaborer et approfondir leurs connaissances dans une vision unitaire de la science et de la technologie » (Guide Matière et matériaux, 1). Dans le cas de l'EIST, la proposition de curriculum intégré s'appuie sur des principes d'unification et d'unité des sciences pour mettre en avant la Science. L'intégration préconisée relève donc ici d'une justification épistémologique et le décloisonnement disciplinaire devient un enjeu d'intelligibilité et de cohérence du savoir enseigné. Un accent est mis sur ce qui est «commun », au risque d'une réduction ou d'une uniformisation, mettant de côté des spécificités et des complémentarités.

Concrètement, la tentative d'EIST s'adosse sur les contenus scientifiques et technologiques des programmes d'enseignement de trois disciplines : Sciences de 
la Vie et de la Terre (SVT), de Technologie (T) et de Physique-Chimie (PC) pour la classe de $6^{\mathrm{e}}$ et de $5^{\mathrm{e}}$. Dans les collèges où l'EIST est expérimenté, une équipe de trois professeurs de chacune des trois disciplines scolaires est mobilisée. Une heure de concertation hebdomadaire, inscrite dans les emplois du temps des professeurs, leur permet d'échanger et de préparer ensemble les séances. Ensuite, chacun d'eux met en oeuvre, individuellement, dans sa classe les séances préparées collectivement. Ainsi, les élèves n'ont plus $1 \mathrm{~h} 30$ de SVT et $1 \mathrm{~h} 30$ de Technologie, comme le prévoient les programmes actuels de la classe de $6^{\text {e }}$, mais $3 \mathrm{~h} 30$ d'un enseignement de Science et Technologie avec un seul professeur' ${ }^{8}$.

Pour accompagner les enseignants, des guides pédagogiques sont mis à disposition sur un site Internet dédié. Le " Guide découverte de l'EIST » présente les caractéristiques de ce nouvel enseignement et deux Guides d'accompagnement proposent une progression pédagogique, des activités pour les élèves et des documents ressources (sur le thème «Matière et matériaux » pour la classe de $6^{\mathrm{e}}$ et sur le thème «Énergie et énergies » pour la classe de $5^{\mathrm{e}}$ ). Ces guides ont été élaborés sous une double tutelle : d'une part, celle de l'Académie des Sciences et de l'Académie des Technologies promoteurs de l'essai d'EIST, d'autre part, celle du MEN pour homologuer la conformité de celui-ci avec les programmes scolaires actuels. Ils représentent une réponse programmatique potentielle à un projet d'enseignement en termes de contenus à enseigner, de progressions, d'activités-élèves, et de propositions pour mettre en œuvre un Enseignement Intégré de Science et de Technologie. Ils résultent à la fois d'une composante prescrite, issue des programmes de chacune des trois disciplines scolaires, et d'une composante novatrice de promotion d'un enseignement désigné comme intégré.

Prenons le cas du Guide «Matière et matériaux ». Celui-ci propose quatre questionnements :

-Qu'y a-t-il autour de nous?

- La matière, de quoi s'agit-il?

- La matière peut-elle changer au cours du temps?

- Comment l'homme utilise-t-il la matière à son profit?

Il présente aussi un découpage en 34 items, explicitement reliés à une ou plusieurs disciplines scolaires : $21 \%$ des items relèvent d'une seule discipline, $42 \%$ de deux disciplines et $37 \%$ de trois. Par exemple :

- l'item «L'eau dans tous ses états » est fléché selon une discipline (Physique-Chimie pour les états de la matière : gaz, liquide, solide) ;

- l'item «Dans le vivant: universalité de la cellule » est fléché selon deux disciplines (SVT pour la cellule, et Technologie pour l'histoire du microscope) ;

- l'item : «Le pain: une transformation sous contrôle» est fléché selon trois disciplines (SVT pour cellule et levure, Physique-Chimie pour les conditions de température et Technologie pour la production de la pâte).

Ce fléchage par disciplines s'adresse aux enseignants. Il leur assure que le Guide respecte bien les «connaissances visées par les programmes disciplinai-

\footnotetext{
${ }^{8}$ Une demi-heure de Physique-Chimie est affectée à l'EIST, il n'y a pas officiellement d'enseignement de PC en classe de $6^{\mathrm{e}}$.
} 
res » (Guide découverte de l'EIST, 29) tout en décloisonnant les disciplines en trois nouveaux champs d'étude : «vivant », «non vivant» et «façonné par l'hom$m e »$. Une reconfiguration des disciplines, envisagée en de nouveaux périmètres d'étude, s'appuie ici sur une activité de discussion, à conduire en classe avec les élèves, concernant le classement des « objets » qui nous entourent : certains sont vivants, d'autres ne le sont pas, d'autres encore sont fabriqués par l'homme. Ce découpage n'est pas strictement superposable aux disciplines scolaires traditionnelles. Si le champ du «vivant» recoupe certes celui des sciences de la vie, il ne fait pas basculer les sciences de la Terre dans le champ du «non-vivant» avec la Physique-Chimie. Seul, le champ du «façonné par l'homme» recoupe la discipline Technologie. Cette reconfiguration en trois «champs» ne valorise pas la connaissance d'objets disciplinaires (la cellule, les états de la matière, l'objet technique, etc.), mais plutôt des thématiques ou de problèmes a-disciplinaires (Loepp 1999 ; Ross 2000). Ainsi, le thème «Eau » est analysé du point de vue :

- «vivant», comme un élément vital des organismes,

- «non-vivant », comme une matière existant sous différents états,

- «façonné par l'homme», comme un agent d'altération des matériaux produit par l'homme.

La proposition d'intégration passe ici par un thème fédérateur. Différentes contributions disciplinaires sont sollicitées pour mettre en œuvre un projet collectif de classe comme, par exemple, «comment fabriquer un dessaleur pour obtenir de l'eau potable à partir de l'eau de mer »?

Il existe, dans le Guide, un seul cas d'intersection de trois champs disciplinaires. À la question «Comment l'homme utilise-t-il la matière à son profit?» correspond l'item «Se nourrir : élevage et culture», avec comme notion essentielle à retenir pour l'élève : "Les espèces végétales et animales, cultivées ou élevées peuvent être considérées comme des objets techniques vivants» (p.60). Le programme officiel de Technologie fait référence au concept d'objet technique et celui de SVT mentionne que l'Homme « est aussi producteur des animaux et des plantes qu'il consomme en les sélectionnant par leurs rendements ou leurs qualités (nutritives, gustatives, etc.) ». Cependant, les plantes cultivées ou les animaux d'élevage ne sont jamais désignés en tant qu'objets techniques vivants dans les programmes de SVT et de Technologie. L'intersection des champs «vivant» et «façonné par l'homme » produit l'émergence d'un nouvel objet d'enseignement : «l'objet technique vivant». Désigner les organismes issus de l'élevage ou de l'agriculture comme des objets techniques vivants signifie qu'ils possèdent à la fois les attributs du vivant et ceux des objets techniques. Par exemple, la modification du vivant ${ }^{9}$ est ainsi assimilée à la production d'un objet technique ayant une fonction précise : répondre à nos besoins alimentaires avec un coût de production et une valeur marchande donnée. De ce point de vue, la vache laitière ou les OGM sont des objets techniques vivants. Ce concept novateur soulève aussi un questionnement éthique auquel répond en partie le Guide EIST en apportant une précision importante : «les espèces végétales et animales, cultivées ou élevées 》 ne

\footnotetext{
${ }^{9}$ Par sélection artificielle au cours de générations ou la production de nouveaux organismes par modification du génome.
} 
sont pas définies de façon absolue comme des objets techniques vivants mais «peuvent être considérées comme des objets techniques vivants». C'est donc le regard et la visée portée sur ces organismes modifiés par l'Homme qui peuvent, ou non, les qualifier d'objets techniques. Bien que cet exemple soit unique, il témoigne d'une possible et nécessaire interaction entre champs face à de nouveaux objets et enjeux d'enseignement.

En résumé, dans les documents institutionnels d'accompagnement l'essai d'EIST, l'intégration apparaît conçue comme :

- une reconfiguration des disciplines en de nouveaux champs disciplinaires (vivant, non-vivant, et façonné par l'homme),

- la construction d'activités pédagogiques autour d'un thème (ex : l'eau) ou d'objets non disciplinaires (ex : le dessaleur), avec contributions des champs disciplinaires,

- le cas d'un nouvel objet d'enseignement : l'objet technique vivant.

\section{2. Échanges entre les enseignants de trois disciplines :}

contribution à un curriculum potentiel

Les concertations, entre les enseignants des trois disciplines, constituent des moments de verbalisation et de réflexion délibérative de l'équipe pour l'élaboration de son projet curriculaire. L'analyse du script de l'enregistrement vidéo d'une concertation (collège A) a permis de saisir différentes pistes envisagées et discutées au sein de l'équipe : les objectifs visés, les méthodes utilisées, les ressources disponibles, etc. La séance de concertation est ici l'occasion, pour l'équipe enseignante, de s'appuyer sur le Guide EIST et/ou le programme de chacune des disciplines, pour les comparer à d'autres ressources disponibles (manuels, sites Internet, etc.), pour échanger et élaborer un curriculum local auto-prescrit.

Des données issues d'un entretien auprès des professeurs (collège A), il apparaît en outre ici, une prise de distance assumée par rapport au Guide et/ou aux programmes. Ainsi, l'enseignant de Technologie explique que l'équipe n'a pas adhéré aux propositions du Guide Matière et matériaux, parce qu'elles leur semblaient contraire à l'idée qu'ils se faisaient d'un EIST (Extrait collège A, tableau 3). Il lui reproche un «saucissonnage » et la juxtaposition des parties des programmes des différentes disciplines.

Q31. - Et tu n'étais pas gênée par des contenus, sur lesquels tu pouvais craindre avoir moins de connaissances et qui t'auraient mis en retrait, vis à vis desquels tu n'aurais pas pu faire face...

R31. - Non, non. Et puis [Silence] [Hésitations]. Mais, bon, il faut dire qu'on a adapté, aussi. On n'a pas fait "matières et matériaux», on a pris "De l'énergie aux matières ». On s'est fait notre propre programme par rapport à ce qui a été proposé.

Q32. - Vous avez fait quelque chose d'indépendant, de différent de ce qui a été proposé par le groupe de pilotage?

R32. - Euh, oui. Parce que l'on n'arrivait pas à rentrer. Parce que la proposition " matières et matériaux », il y a avait 4 ou 5 grands points et c'était du saucissonnage : il y avait physique, il y avait SVT, il y avait techno avec matériaux. Bon, on voyait les matériaux au travers d'une voiture avec le recyclage de la batterie, par exemple. Tout ça, sincèrement, on n'a pas pu aborder ça comme ça. C'était... Tu sentais que c'était des compétences, des parties de programme qui étaient juxtaposées, euh...

Tableau 3 : Extrait de l'entretien avec le professeur de Technologie du collège A 
L'enregistrement de séance de concertation entre les trois enseignants porte sur le thème «Énergie et moyens de transport ». Un mince fil relie la discussion avec le programme de Technologie de la classe $6^{\mathrm{e}}$ (nature des énergies utilisées pour le fonctionnement de moyens de transports). L'analyse des échanges permet de considérer 8 points ayant fait objet de discussion : la définition de l'énergie, le choix des activités pour les élèves, les difficultés des élèves, les compétences attendues des élèves, le choix du matériel à utiliser, la progression pédagogique, le planning des séances et la répartition des tâches au sein de l'équipe. L'essentiel du temps de discussion est consacré à la progression pédagogique $(35 \%)$ et au choix du matériel à utilisé (37\%). C'est aussi l'occasion d'échanger sur la notion d' « énergie », sur les façons de l'expliquer et de l'illustrer auprès des élèves (Extrait collège $\mathrm{A}$, tableau 4 ).

SVT 1 : Les énergies renouvelables, énergie et énergie renouvelable. Pour voir le concept d'énergie renouvelable, il faut voir énergie d'abord. Pour démarrer la leçon, on démarre de quoi? De problèmes, de petites choses qu'ils peuvent voir à la télé... de vidéo ? Du journal TV...

TECH 2- Oui, des images, c'est bien... (...)

SVT 5... On démarre sur le transport ? (...)

PHYS 17 : Qu'est-ce qui fait bouger les moyens de transports? (...)

TECHN 21 : Qu'est-ce qui permet... qu'est-ce qui met en mouvement ? (...)

PHYS 27 : Il y a une transformation de l'énergie (...)

SVT 35 : Pour faire bouger (...)

PHYS 53 : Ce qui nous intéresse, c'est énergie dans le sens de faire bouger quelque chose. Ce n'est pas l'idée de transformation, pas pour l'instant. (...)

TECHNO 56 : Prends la patinette, comme ça t'es pas embêté. T'as pas d'électricité, t'as rien. Comme ça, ça met quand même l'engin en mouvement donc, euh...

SVT 57 : Et puis on aura une source d'énergie différente. On aura l'énergie musculaire. On peut aller... patinette, vélo...(...)

PHYS 106: C'est le mot énergie dans l'expression énergie de mouvement. Pour eux (les élèves), c'est pas de l'énergie çà. (...)

PHYS 109: Tu peux aussi dire que l'énergie se transforme en chaleur. Au mieux, au pire, c'est...

SVT 110: Après, le mouvement, c'est aussi une énergie qui peut servir à autre chose. J'ai peur qu'ils ne comprennent pas bien...

TECH 111 : Après, tu as des consomma...(Interrompu).

PHYS 112: Je pense que tu vas plus loin que tu...

TECH 113 : Il faut rester sur énergie de mouvement, énergie mécanique mais là... PHYS 114 : Non, pas mécanique.C'est un piège et...

TECH 115: Ca va embrouiller. Quand ils (élèves) apprennent une définition, quand on travaillait sur les cellules, c'est des mots, c'est tout. Il faut quand même bien qu'ils utilisent aussi les termes disponibles, on ne les a pas inventé, ces termes-là. Donc ça, c'est une synthèse.

Tableau 4 : Extrait de la séance de concertation entre les trois enseignants du collège $\mathrm{A}$ (Physique-Chimie, SVT, Technologie)

L'enseignant de SVT utilise ici l'expression «énergie musculaire », sans que cette notion interroge les deux autres enseignants sur la nature de l'énergie (chimique, mécanique, calorifique). En ce qui concernent les échanges sur «les énergies renouvelables » : pour l'enseignant de Physique-Chimie, celles-ci n'exis- 
tent pas car l'énergie ne disparaît pas, seule, la source d'énergie peut s'épuiser, tandis que l'enseignement de Technologie considère que cette distinction n'est pas jugée très utile à donner aux élèves. La collaboration entre enseignants ne règle pas ici tous les arbitrages. C'est la consultation de manuels scolaires ou de sites internet, voire la détermination d'un enseignant, qui fait aussi autorité. Chacun des enseignants avance en outre des suggestions autour du matériel à proposer aux élèves : une occasion de faire découvrir et partager à l'équipe, la manipulation d'instruments spécifiques à sa discipline : microscope, batterie, pile, capteur solaire, etc.

En résumé, lors de cette séance de concertation, l'équipe enseignante se détermine en fonction de plusieurs pistes possibles. Elle consulte dans un premier temps les Guides EIST, les programmes scolaires, mais aussi les manuels et les sites Internet. Elle les compare et les met en discussion pour en évaluer la pertinence dans le cadre de son propre projet éducatif. Tout en prenant en compte les recommandations institutionnelles, elle s'en distancie pour le repenser, s'interroger concrètement sur « le comment faire » et sur les objectifs qu'elle préfère assigner à un EIST. Différentes propositions sur l'organisation, la progression des leçons, et les activités des élèves sont envisagées comme contributions à un curriculum potentiel. Cette approche réflexive s'accompagne, progressivement, d'une élaboration curriculaire selon les besoins, les contraintes et les objectifs locaux. En conséquence, au fur et à mesure des concertations le champ potentiel se réduit en fonction des choix, des sélections, des réaménagements, des ajouts ou des retraits de certains contenus et/ou modalités envisagés.

Les concertations représentent aussi des moments où l'équipe enseignante arrête ses choix parmi les propositions énoncées. Elle décide, par exemple, de produire un document de travail que chaque enseignant aura à suivre. Ainsi, l'équipe enseignante élabore peu à peu un curriculum local auto-prescrit, indiquant ses objectifs, sa progression, les activité-élèves, le matériel utilisé pour la mise en œuvre d'EIST.

\subsection{L'élaboration d'un curriculum local auto-prescrit}

L'équipe EIST du collège $\mathrm{B}$ a mis à notre disposition sa progression sur les deux premiers trimestres (élaborée lors de séances de concertation avant la rentrée scolaire), ainsi que les fiches des séances et des activités-élèves. L'architecture globale de ce curriculum auto-prescrit est guidée ici par un canevas narratif où l'équipe enseignante met en scène un jeune aventurier, parti à la recherche d'une île mystérieuse qui contient un trésor. Au cours de cette aventure, il est amené à utiliser différents moyens de transport pour se déplacer, à se servir des appareils de mesures pour s'orienter, à cultiver des plantes et à élever des animaux pour se nourrir. Ce curriculum auto-prescrit s'organise ici autour de thèmes (cycle de l'eau, reproduction des plantes, construction d'un vivarium,...) et de résolutions de problème (Comment un dirigeable peut-il voler? Quelles sont les conditions de germination des graines ?...)

Un extrait de ce curriculum auto-prescrit (tableau 5) fait apparaitre un découpage en «thème », "problème », «compétences », «activités » et une référence aux disciplines qui ont été élaborés par l'équipe elle-même (et non par les chercheurs). Les compétences y sont systématiquement associées à une discipline 
(SVT, Technologie ou PC). Les activités correspondent ici à une mise en situation du respect de consignes (repérer, indiquer, nommer, classer, etc.).

La catégorisation, choisie ici par l'équipe, constitue un indicateur de leur appropriation du «I $»$ de l'EIST. Sur les 8 thèmes du curriculum auto-prescrit :

- un thème, «Les objets », présente l'association de trois disciplines (Tech, SVT et PC) ;

- deux thèmes, «Découvertes des appareils de mesures» et «Le cycle de l'eau », associent deux disciplines (SVT et PC) ;

- deux thèmes, «Les conditions de vie des êtres vivants» et «Les conditions de germination des graines », relèvent des SVT ;

- deux thèmes, «Le fonctionnement des objets » et «La découverte des matériaux », relèvent de la Technologie.

\begin{tabular}{|c|c|c|c|}
\hline Thème & Problème & Compétences & Activités \\
\hline \multirow{10}{*}{$\begin{array}{l}\text { Les objets } \\
\text { (Technologie, } \\
\text { SVT, PC) }\end{array}$} & $\begin{array}{l}\text { Quels sont les éléments } \\
\text { qui constituent le dirigea- } \\
\text { ble? }\end{array}$ & $\begin{array}{l}\text { (Tech) Identifier les princi- } \\
\text { paux éléments qui consti- } \\
\text { tuent l'objet }\end{array}$ & $\begin{array}{l}\text { repérer, nommer les élé- } \\
\text { ments (du dirigeable) }\end{array}$ \\
\hline & $\begin{array}{l}\text { Quels sont les objets né- } \\
\text { cessaires pour son } \\
\text { voyage? }\end{array}$ & $\begin{array}{l}\text { (Tech) Associer un usage à } \\
\text { un besoin }\end{array}$ & $\begin{array}{l}\text { indiquer les objets nécessai- } \\
\text { res au voyage }\end{array}$ \\
\hline & $\begin{array}{l}\text { Comment classifier les } \\
\text { objets qui nous entou- } \\
\text { rent? }\end{array}$ & $\begin{array}{l}\text { (Tech) Identifier objet natu- } \\
\text { rel et objet technique }\end{array}$ & $\begin{array}{l}\text { classer les objets en vivant } \\
\text { et non vivant }\end{array}$ \\
\hline & $\begin{array}{l}\text { Comment classifier les } \\
\text { objets qui nous entou- } \\
\text { rent? (suite) }\end{array}$ & $\begin{array}{l}\text { (SVT) Distinguer le Vivant } \\
\text { et le Non Vivant }\end{array}$ & classer \\
\hline & $\begin{array}{l}\text { Qu'est-ce qu'un environ- } \\
\text { nement? }\end{array}$ & $\begin{array}{l}\text { (SVT) Identifier les relations } \\
\text { entre les êtres vivants }\end{array}$ & \\
\hline & \multirow[b]{2}{*}{$\begin{array}{l}\text { L'homme peut agir sur } \\
\text { son environnement }\end{array}$} & $\begin{array}{l}\text { (SVT) Identifier les compo- } \\
\text { santes de l'environnement }\end{array}$ & réaliser un tableau \\
\hline & & $\begin{array}{l}\text { (SVT) Identifier des trans- } \\
\text { formations apportées par } \\
\text { l'Homme dans l'environne- } \\
\text { ment. }\end{array}$ & citer les événements \\
\hline & \multirow{2}{*}{$\begin{array}{l}\text { Pour quels usages sont } \\
\text { fabriqués les objets tech- } \\
\text { niques? }\end{array}$} & $\begin{array}{l}\text { (Tech) Identifier la fonction } \\
\text { d'usage d'un objet. }\end{array}$ & $\begin{array}{l}\text { indiquer la fonction de cha- } \\
\text { que objet }\end{array}$ \\
\hline & & $\begin{array}{l}\text { Savoir retrouver des infor- } \\
\text { mations dans un texte ou sur } \\
\text { internet }\end{array}$ & $\begin{array}{l}\text { rédiger les réponses aux } \\
\text { questions posées }\end{array}$ \\
\hline & $\begin{array}{l}\text { Comment un dirigeable } \\
\text { peut-il voler? }\end{array}$ & $(P C)$ La masse d'un gaz & $\begin{array}{l}\text { réaliser une démarche scien- } \\
\text { tifique }\end{array}$ \\
\hline
\end{tabular}

Tableau 5 : Extrait du curriculum auto-prescrit du collège $B$

reconstruit à partir des préparations communes et des fiches d'activité pour les élèves.

(Tech : Technologie ; PC : Physique-Chimie ; SVT : Sciences de la Vie et de la Terre)

Nous pouvons noter, sur les 21 items du curriculum auto-prescrit du collège $\mathrm{B}$, une forme de «mono-disciplinarité » en SVT (43\%) et en Technologie (33\%), une association SVT et PC (14\%), Tech et PC (5\%). D'autres associations telles, Tech/SVT ou Tech/SVT/PC sont ici absentes.

Ces enseignants du collège $\mathrm{B}$ ont, par ailleurs, choisi de commencer leur enseignement par une présentation, élaborée en commun, de l'EIST aux élèves. «Vous êtes en Classe Sciences. Qu'est-ce que la Classe Sciences? Nous allons nous retrouver 4 heures par semaine pour vous enseigner des Sciences : des SVT, 
de la Technologie et de la Physique Chimie. Un seul enseignant se chargera de ces trois disciplines, il sera votre professeur de Sciences durant toute l'année (...). Notre but est de vous confronter à différents problèmes scientifiques que vous tâcherez de résoudre avec l'aide de votre professeur de Sciences ». La dénomination «Sciences» (au pluriel ici) recouvre, dans ce cas, sciences expérimentales et technologie, avec un et qui peut apparaître comme inclusif, considérant la Technologie plutôt comme science appliquée.

Nous avons, en outre, constaté que l'élaboration d'un curriculum autoprescrit ne relevait pas totalement d'un travail collectif ou collaboratif. Chacune des équipes des deux collèges a dévolu, à chaque professeur spécialiste, la rédaction d'une partie de séance considérée comme relevant de sa discipline. Par exemple dans le collège $\mathrm{A}$, le choix et l'organisation des séances sur «énergie» sont décidés collectivement, mais il est laissé au professeur de SVT le soin d'élaborer et de rédiger ce qui concerne l'énergie musculaire, au professeur de PC la transformation de l'énergie, et au professeur de technologie l'utilisation de l'énergie.

\subsection{Des curriculums co-produits}

Chaque enseignant met en œuvre, de façon continuée tout au long de l'année scolaire, le curriculum auto-prescrit élaboré par l'équipe, en l'adaptant à sa classe, en fonction du questionnement des élèves et selon son habitus. Prenons le cas de l'enseignement de l'énergie dans le collège $\mathrm{A}$, dont nous avons présenté auparavant une pré-analyse (Fortin, Lasson et Coquidé 2013). Le professeur de Technologie indique aux élèves que «l'objet technique a besoin d'énergie pour avancer, pour fonctionner », "pour fonctionner, la voiture a besoin d'essence. Elle consomme de l'essence». Il pointe le carburant «essence». Dans la classe d'EIST du professeur de Technologie ici, l'énergie est considérée comme transformée mais également comme consommée et, par conséquent, elle peut disparâ-tre. Il s'agit là d'un point de vue qui se distingue particulièrement de celui développé dans la mise en oeuvre d'EIST du professeur de Physique-Chimie, qui exprime un principe de conservation de l'énergie et insiste auprès de ces élèves : " Il y a une chose qu'il faut savoir, l'énergie ne disparaît pas. L'énergie qui est là depuis le début de l'univers est là, elle ne disparaît pas. Alors, qu'est-ce qu'elle est devenue si elle ne disparaît pas? Elle brûle, elle se transforme. L'énergie, elle ne disparaît pas, elle se transforme. Il y a une transformation d'énergie en énergie de mouvement ». Nous pouvons considérer ici un effet «spécialisation disciplinaire » dans le curriculum co-produit d'EIST de chacun de ces professeurs.

Par ailleurs, en classe d'EIST, l'enseignant peut parfois être conduit à enseigner un sujet sans aucun lien avec sa spécialité et sa formation. Par exemple, dans le collège $\mathrm{B}$, une séance porte sur la fécondation des plantes à fleur. Elle a été préparée en concertation et le professeur de SVT a rédigé une fiche descriptive des organes reproducteurs d'une fleur pour expliquer à ses collègues les modalités de la fécondation. Cette fiche est également distribuée aux élèves pendant la séance d'EIST. En classe, le professeur de Physique-Chimie dessine et légende, au tableau, une fleur puis décrit différentes étapes (pollinisation, germination, et rencontre des gamètes). Deux des légendes écrites au tableau sont cependant fausses et l'explication de la fécondation est erronée ${ }^{10}$. Certains élèves remarquent que les

\footnotetext{
${ }^{10}$ Le professeur de PC explique que le grain de pollen tombe dans le style, comme dans un tobog-
} 
légendes du dessin au tableau ne concordent pas avec celles du schéma de la fiche distribuée. La fiche distribuée aux élèves donne la possibilité à l'élève «d'interroger » ou de «contredire» le professeur en confrontant «ce qu'il dit» avec «ce qui est écrit ». C'est aussi une balise pour l'enseignant. Ni la fiche, ni les échanges préalables avec le collègue de SVT, n'ont cependant permis au professeur de PC de maîtriser le contenu en jeu. Une connaissance opératoire de l'anatomie et la biologie des plantes à fleur est nécessaire pour distinguer la germination du grain de pollen de la fécondation. Dans ce cas précis, l'enseignant ne dispose pas d'une autonomie didactique suffisante lui permettant de répondre avec justesse aux interrogations des élèves. Il se trouve en difficulté au point de ne plus être en mesure de conduire l'apprentissage des élèves, faute de pouvoir répondre à leur questionnement, de valider ou d'infirmer leurs propositions, avec des conséquences dans la co-production curriculaire, continuée sur toute l'année scolaire.

\section{BILAN ET DISCUSSION}

Dans notre étude, nous avons constaté que le projet d'un Enseignement Intégré de Science et Technologie n'était pas défini à l'avance. Chaque équipe a utilisé cette possibilité d'ouverture curriculaire et a élaboré, progressivement et plus ou moins collectivement, un curriculum auto-prescrit, en particulier lors des séances de concertation. Ces temps d'échanges et de négociations ont contribué à la construction progressive d'un projet curriculaire partagé s'appuyant sur :

- les savoirs et/ou des pratiques de spécialités disciplinaires déjà existantes,

- une collaboration entre collègues pour clarifier, expliciter les contenus à enseigner et formuler des objectifs communs,

- une formation, issue de cette collaboration, pour utiliser en classe du matériel biologique, technologique et/ou physico-chimique à manipuler avec les élèves. Si riche soit cette collaboration entre collègues de différentes disciplines, elle n'a pas permis, au vu de cette étude de cas d'observer une réelle co-formation, aboutissant à une prise en charge totalement autonome de chaque enseignant en classe d'EIST.

Cette auto-prescription s'accompagne aussi d'une expérience concrète de l'altérité disciplinaire. Dans nos observations, une forme de reconfiguration curriculaire est constatée et se joue, dans un rapport entre la spécialité disciplinaire de chaque enseignant et la rencontre de celles d'autres enseignants de disciplines différentes, et une mise en relation entre différentes disciplines scientifiques et technologiques pour envisager une forme d'intégration. Dans notre étude, nous avons constaté, par rapport à un ensemble de curriculums possibles d'EIST, une élaboration de formes principalement de juxtaposition disciplinaire ou de pluridisciplinarité. La démarche pédagogique d'investigation, mise en avant dans le projet institutionnel de l'essai d'EIST, n'est, en outre, jamais apparue centrale ici, ni dans le

gan, pour rejoindre l'ovule dans l'ovaire, confondant ainsi la cellule sexuelle avec le grain de pollen. [Le grain de pollen produit au niveau des étamines est transporté par le vent ou les insectes sur le stigmate de la fleur, c'est la pollinisation. Il germe en formant un long tube pollinique qui contient le gamète mâle, c'est la germination du tube pollinique. Celui-ci s'enfonce dans le style, structure entre le stigmate à l'ovaire, jusqu'à l'ovaire et rencontre l'ovule : c'est la fécondation]. 
curriculum auto-prescrit élaboré, ni dans le discours des enseignants. Par ailleurs, les écarts entre curriculum auto-prescrit et curriculums co-produits mettent en évidence certaines difficultés des deux équipes enseignantes. Nous pouvons remarquer que le déficit d'autonomie des enseignants en classe d'EIST s'explique non pas tant par un déficit de connaissances académiques ou pédagogiques, mais plutôt par l'absence d'une technicité partagée.

Ces constats conduisent à envisager, dans une perspective de formation, certaines nécessités, pour aider une équipe d'enseignants à problématiser une reconfiguration de curriculum en EIST. Pour élaborer un curriculum auto-prescrit, l'équipe enseignante pourrait problématiser les enjeux éducatifs d'un enseignement intégré et envisager divers curriculums possibles. Les aides institutionnelles et les formations actuelles ne semblent pas remplir, actuellement, cette fonction. En effet, le nouveau découpage en champs disciplinaires (vivant, non-vivant, façonné par l'homme), les nouvelles pratiques de référence issues de la vie quotidienne et l'appel à démarche d'investigation qui y sont avancés ne suffisent pas à tisser des liens, à aider réfléchir aux convergences et aux complémentarités disciplinaires. L'interdisciplinarité, fortement revendiquée dans les recommandations institutionnelles, ne peut, d'un point de vue de recherche, être réduite à ce qui est commun aux différentes disciplines ou à des outils méthodologiques. Elle est d'abord une mise en relation, par complémentarité ou par coordination, des disciplines (Astolfi 2008). Les outils conceptuels que nous avons mobilisés pourraient contribuer à cette médiation et favoriser la réflexion des équipes, en particulier ceux sur les formes d'intégration ou d'indifférenciation disciplinaires, ceux sur les questionnements et les élaborations curriculaires. La rétroaction, au niveau de chaque équipe, des curriculums co-construits sur le curriculum auto-prescrit pourrait aussi favoriser l'exploration d'un curriculum potentiel.

\section{POUR ÉLARGIR LE DÈBAT : DES CONTENUS D'ENSEIGNEMENT ET DES ARTICULATIONS DANS UN SYSTÈME CURRICULAIRE}

D'un côté, les modalités réalisées de l'enseignement, plus ou moins flexibles, sont à la fois sources et résultats de tensions, avec une prégnance de forme scolaire (Vincent et alii 1994), visibles par exemple dans l'organisation qui est privilégiée (par exemple organisation en classe et/ou en dispositif intra ou interclasse). D'un autre côté, les contenus de l'enseignement secondaire, avec les visées de valeurs, de savoirs, de cultures et pas seulement en termes de compétences évaluables qu'ils permettent, sont porteurs de multiples enjeux éducatifs (Gauthier 2006, Boyer et Reuter 2012). Envisager les apports de l'enseignement en termes de contenus évite en effet de se restreindre à des savoirs, le plus souvent discursifs en France (un texte de savoir). «Savoir» dans la perspective d'une éducation scientifique ce peut, par exemple, savoir clarifier les situations problématiques, savoir rechercher les données nécessaires, savoir mobiliser les connaissances construites pour trouver des réponses possibles aux problèmes... Une perspective de contenus d'enseignement permet, en outre, d'envisager une certaine mise à distance avec des disciplines scolaires déjà constituées et leurs dépendances à l'égard 
de disciplines académiques intervenant dans la formation des enseignants (Martinand 2003b, White 2004).

Alors, que faire des disciplines scolaires pour l'enseignement scientifique et technique au collège ? Les questions de structuration et d'organisation disciplinaires restent toujours sous-jacentes, en particulier par les aspects de recrutement et de formation d'enseignants. Les disciplines scolaires ne sont cependant pas immuables (Coquidé 2008). Martinand (2003a) souligne que les disciplines du collège sont des montages, des compromis, des constructions historiques plus ou moins rationnelles, pour prendre en charge certaines des missions affectées au collège pour une certaine durée. Elles sont portées par les corps sociaux que sont les enseignants dont l'exercice quotidien du métier infléchit grandement les prescriptions administratives. D'un point de vue épistémologique, le terme discipline ne désigne pas seulement un domaine délimité de la connaissance, mais aussi l'obligation de « discipliner l'esprit », afin d'entrer dans une certaine modélisation théorique allant contre le sens commun et de développer des méthodologies spécifiques. Celles-ci peuvent être approchées comme des manières d'interroger ou d'intervenir sur le monde avec des démarches, des méthodes, des instruments et des concepts, parfois communs, parfois spécifiques. La façon d'appréhender l'énergie, par exemple, n'est pas la même dans une approche technologique (la consommation de l'énergie) et dans une approche physicienne (la conservation de l'énergie). La chimie des éprouvettes et des équations bilan diffère de la chimie biologique des compartiments cellulaires et des cycles. Ce sont des processus, de scolarisation et de «disciplinarisation », des constructions et des normalisations sociales qui conduisent à un ensemble structuré et structurant, avec principe de progressivité d'une matière scolaire et cohérence d'ensemble (Coquidé 2008). Une interdisciplinarité ne saurait donc nier les disciplines ni les transcender. Argumentant la «saveur des savoirs », Astolfi (2008) souligne la signification des savoirs et des disciplines, pour les professeurs d'une part, pour les élèves d'autre part, et la nécessité de pouvoir caractériser les spécificités disciplinaires. La «conscience disciplinaire », avancée par Reuter (2007), peut représenter un outil conceptuel pour étudier la manière dont les élèves construisent, peu à peu, ces disciplines scolaires.

La tentative d'EIST n'apparaît actuellement pas comme un «prototype » de nouvelle discipline scolaire. Dans son élaboration et dans ses mises en œuvre, cet essai pourrait davantage être rapproché de dispositifs de croisement de disciplines scolaires, tels les Travaux Personnels Encadrés (TPE) en lycée (Schneeberger et alii 2004, Fortin 2004) ou les Itinéraires de découvertes (IDD) au collège (Magneron et Lebeaume 2004). Ces dispositifs résultent d'une rencontre entre le thème choisi par les élèves et/ou les enseignants, et les connaissances des enseignants sur ce thème. De cette rencontre émergent différents modes de fonctionnement, de collaborations et d'interactions entre les enseignants et les élèves. Les analyses qui y ont été conduites, sur les postures enseignantes, sur les interactions entre enseignants et élèves, ou sur la place de l'interdisciplinarité ont montré que si des convergences entre disciplines étaient possibles, elles n'effaçaient pas l'irréductible spécificité de chacune d'elles. À ce titre, l'essai d'EIST peut aussi être considéré comme dispositif « émergeant », résultant d'une tension entre des contenus disciplinaires inscrits dans les programmes scolaires, des recommandations institutionnelles et des projets d'équipes locaux. De cette rencontre locale de spé- 
cialistes disciplinaires émergent différents modes de fonctionnement, de collaborations et d'interactions entre les enseignants. Les élaborations et les constructions curriculaires que nous avons analysées témoignent d'une innovation professionnelle, dans laquelle les enseignants ont fait preuve de créativité et de collaboration, en concevant un curriculum auto-prescrit.

Un projet de transformation ou d'élaboration curriculaire nécessite de penser et de tenir tout un ensemble (Martinand 2012) : les missions et les enjeux éducatifs, le pilotage (par les contenus, par les objectifs ou les compétences, par les expériences) (Ross 2000), l'organisation (par exemple dispositif et/ou discipline...), la sélection et l'élaboration de contenus, la programmation, la réalisation d'aides pédagogiques et la formation dans une nouvelle cohérence. Pour un travail de renouvellement et de restructuration des contenus des curriculums scientifiques et technologiques du secondaire, Martinand (2003 a) propose de considérer tout un système curriculaire (disciplines, actions éducatives non disciplinaire, moments d'intégration pluridisciplinaires) à penser et à articuler, avec des distinctions fortes, des relations d'opposition mais également de complémentarité, et des dispositifs de coopération et de coordination.

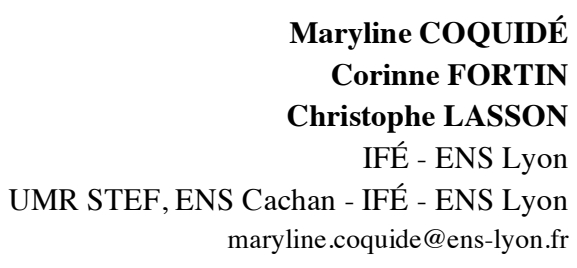

Abstract : The test of an Integrated Teaching of Science and Technology (EIST) in some French middle school aims to change the organization of distinct scientific and technical school disciplines (biology and earth sciences, physical sciences, technology). With a didactical theorisation of the curriculum (Martinand 2003a, 2012), we analyze in situ the dynamic curriculum design and implementation of EIST by teaching teams of two colleges. The two case studies show rather a juxtaposition of disciplinary contributions than integration.

Keywords : school discipline, device, curriculum, science, technology, curricular transformation.

\section{Bibliographie}

Astolfi J.-P. (2008) La saveur des savoirs. Disciplines et plaisir d'apprendre. Paris : ESF.

Baillat G. \& Niclot D. (2003) «Les enseignants généralistes et les enseignants spécialistes face à l'intégration des savoirs » - in: Y. Couturier (coord.) Dossier thématique La condition interdisciplinaire du travail. Esprit Critique 5 (1).

http://194.214.232.113/Dossiers/index_auteurs_archives.asp\#Baillat_Gilles

Bernstein B. (1975) Langage et classes sociales. Codes sociolinguistiques et contrôle social. Paris : Les Éditions de Minuit. 
Boyer C. \& Reuter Y. (2012) (coord.) Les contenus disciplinaires - Recherches en Didactiques 13 .

Chevallard Y. (2004) «Vers une didactique de la codisciplinarité. Notes sur une nouvelle épistémologie scolaire»-Texte pour les Journées de didactique comparée, Lyon, 3-4 mai.

http://yves.chevallard.free.fr/spip/spip/article.php3?id_article=45

Coquidé M. (2001) Les parcours sciences expérimentales et technologiques, séminaire de l'académie de Paris.

http://parcours-diversifies.scola.ac-paris.fr/parcourssciences/pratiques_experimentales.htm

Coquidé M. (2008) «Les disciplines scolaires et leurs enseignants spécialisés : distinguer pour pouvoir articuler et travailler ensemble »- in : A. Hasni et J. Lebeaume (dir.) Interdisciplinarité et enseignement scientifique et technologique (51-74). Sherbrooke: Éditions du CRP et Lyon : INRP.

Coquidé M., Fortin C. \& Rumelhard G. (2009) «L'investigation : fondements et démarche, intérêts et limites »-Aster 49 (51-78).

Coquidé M., Fortin C. \& Lasson C. (2011) Étude sur l'élargissement de la spécialité enseignante dans l'enseignement intégré de science et de technologie (EIST) au collège. Rapport final de recherche STEF-IFE, 63 p. http://www.stef.ens-cachan.fr/docs/eist_rapport_stef_ife_dec_2011.pdf

Dell'Angelo M., Coquidé M. \& Magneron N. (2011) «Statut de l'investigation dans des standards de l'enseignement scientifique : cas des USA, de la Suisse et de la France » - in : B. Calmettes (dir.) Démarches d'investigation : références, représentations, pratiques et formation. $\mathrm{Pa}-$ ris : L'Harmattan.

Dutercq Y. \& Derouet J-L. (2004) Le collège en chantier. Lyon : INRP.

Fensham P.J. (2002) «De nouveaux guides pour l'alphabétisation scientifique » Revue Canadienne de l'Enseignement des Sciences, des Mathématiques et des Technologies 2, 2 (133-149).

Ferry L. (1995) «Qu'apprendre au collège ?» - Le Débat 87 (147-180).

Forquin J-C. (2008) Sociologie du curriculum. Rennes : PUR.

Fortin C. (2004) « Travaux personnels encadrés ou l'effet causal de l'interdisciplinarité »-Aster 39 (61-90).

Fortin C., Lasson C. \& Coquidé M. (2013) «Une étude de cas de l'enseignement de l'énergie : variations de contenus dans l'Enseignement Intégré de Science et Technologie au collège » - in : B. Daunay, Y. Reuter et A. Thépaud (éds.) Les contenus disciplinaires. Approches comparatistes (37-50). Lille : Septentrion.

Gauthier F.-R. (2006) Les contenus de l'enseignement secondaire dans le monde. Paris : Unesco.

http://unesdoc.unesco.org/images/0014/001475/147570f.pdf

Jenkins E. W. (2009) « Reforming school science education : a commentary on selected reports and policy documents »-Studies in Science Education $45,1(65-92)$.

Jodelet D. (1989) Les représentations sociales. Paris : PUF.

Lebeaume J. (2002) «De quelques enseignements expérimentaux... disparus » Cahiers Pédagogiques 409 (11-14). 
Lebeaume J. \& Magneron N. (2004) «Itinéraires de découverte au collège : à la recherche des principes coordinateurs »-Revue Française de Pédagogie 148 (101-118).

Lebeaume J. (2008) «Les sciences et la technologie dans l'enseignement obligatoire : curriculums et spécialités enseignantes »- in : A. Hasni et J. Lebeaume (dir) Interdisciplinarité et enseignement scientifique et technologique (33-49). Sherbrooke: Éditions du CRP et Lyon: INRP.

Lebeaume J. (2009) «Les travaux scientifiques expérimentaux pour les classes de $6^{\text {e }}$ et de $5^{\text {ème }}$. Retour sur les premiers essais d'un enseignement expérimental »-Aster 49 (25-50).

Lenoir Y. (2006) « Practices of disciplinarity and interdisciplinarity in Quebec elementary schools : Results of twenty years of research »-Journal of Social Science Education 4-2006. http://www.jsse.org/2006/2006-4

Lenoir Y., Larose F. \& Lessard C. (2006) « La structuration par domaines du nouveau curriculum de l'enseignement primaire : analyse critique du modèle »- in : Y. Lenoir, F. Larose et C. Lessard (éds.) Le curriculum de l'enseignement primaire: regards critiques sur ses fondements et ses lignes directrices (170-200). Sherbrooke: Éditions du CRP.

Lenoir Y. (2008) «L'interdisciplinarité dans l'enseignement scientifique : apports à privilégier et dérives à éviter « - in : A. Hasni et J. Lebeaume (éds.) Interdisciplinarité et enseignement scientifique et technologique (1732). Sherbrooke : Éditions du CRP et Lyon : INRP.

Lessard C. \& Barrère A. (dir.) (2005) Travailler ensemble? Des réformes éducatives aux pratiques enseignantes. Lyon : INRP.

Loepp F.L. (1999) « Models of curriculum integration » - Journal of Technology Studies XXV, 2 (21-25).

Magneron N. \& Lebeaume J. (2004) «Les élèves et les itinéraires de découverte : entre temps extraordinaire et postures moins ordinaires »-Aster 39 (153-172).

Marcel J.-F, Dupriez V., Perisset Bagnoud D. \& Tardif M. (dir) (2007) Coordonner, collaborer, coopérer. De nouvelles pratiques enseignantes. Bruxelles : De Boeck Université.

Martinand J.-L. (1994) «La didactique des sciences et de la technologie et la formation des enseignants »-Aster 19 (61-75).

Martinand J.-L. (2003a) «L'éducation technologique à l'école moyenne en France : problèmes de didactique curriculaire »-La Revue Canadienne de l'Enseignement des Sciences, des Mathématiques et des Technologies 3, 1 (100-116).

Martinand J.-L. (2003b) « Missions et fonctions des disciplines dans l'enseignement au collège »- in : J.-L. Derouet (dir.) Le collège unique en question (183-189). Paris : PUF.

Martinand J.-L. (2012) Éducation au développement durable et didactiques du curriculum. Conférence XIX $X^{e}$ Colloque AFIRSE, Lisbonne. 
Martinand J.-L. (2013) «Pour une posture didacticienne, comparatiste et proactive collège » - in : B. Daunay, Y. Reuter et A. Thépaud (éds.) Les contenus disciplinaires. Approches comparatistes (247-256). Lille : Septentrion.

Mérini C. (2005) Travail conjoint et professionnalité enseignante. Paris : ADASE.

Mons N., Pons X. \& Behrens M. (2006) Les standards en éducation dans le monde francophone : une analyse comparative. Neuchatel : Institut Romand de Recherches et de Documentation Pédagogiques.

Perrenoud P. (1993) «Curriculum : le formel, le réel, le caché » - in : J. Houssaye (dir.) La pédagogie : une encyclopédie pour aujourd'hui (61-76). Paris : ESF.

Reuter Y. (2007) «La conscience disciplinaire, présentation d'un concept»Éducation \& Didactique 1, 2 (57-71)

Rocard M., Csermely P., Jorde D., Lenzen D., Walberg-Henrikisson H. \& Hemmo V. (2007) L'enseignement scientifique aujourd'hui : une pédagogie renouvelée pour l'avenir de l'Europe. Commission Européenne.

Ross A. (2000) Curriculum : Construction and Critique. London \& New York: Falmer Press.

Rumelhard G. \& Desbeaux-Salviat B. (2000) « Rencontres entre les disciplines » - Aster 30 (1-7).

Schneeberger P., Cotten A., Goix H, Goix M., Rodriguez R. \& Vidal M. (2004) « Types de travaux personnels encadrés, postures d'enseignants et structuration »-Aster 39 (39-60).

Vincent G., Lahire B. \& Thin D. (1994) L'éducation prisonnière de la forme scolaire. Scolarisation et socialisation dans les sociétés industrielles. Lyon : PUL.

Wallace J., Sheffield R., Rennie L. \& Venville G. (2007) « Looking back, looking forward: Re-searching the conditions for curriculum integration in the middle years of schooling » - The Australian Educational Researchers 34, 2 (29-49).

White J. (dir.) (2004) Rethinking the School Curriculum. Values, Aims, and Purposes. Londres : Routledge Falmer. 